This is a provisional PDF only. Copyedited and fully formatted version will be made available soon.

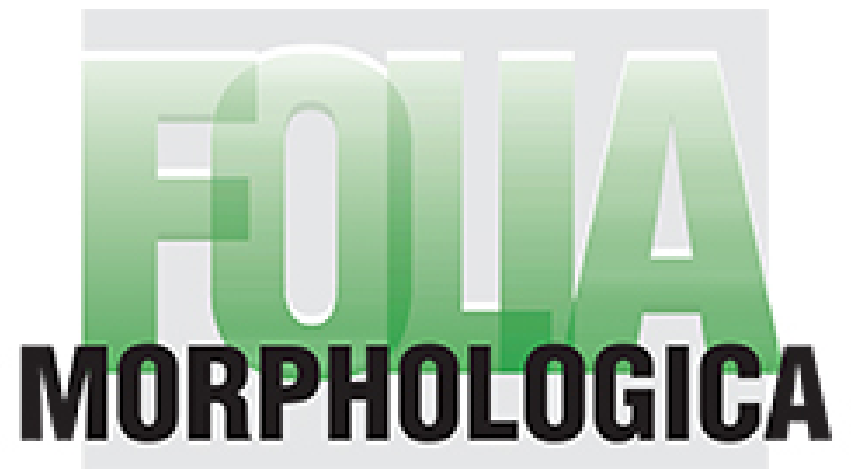

ISSN: 0015-5659

e-ISSN: $1644-3284$

\title{
The relationship between the aggrecan VNTR polymorphism and its content in lumbar intervertebral discs
}

Authors: P. Pękala, D. Felkle, K. Dykas, A. Jarosz, P. Elnazir, T. Konopka, J. A. Walocha, J. Dulinska-Litewka

DOI: 10.5603/FM.a2022.0019

Article type: Original article

Submitted: 2022-02-07

Accepted: $2022-02-09$

Published online: 2022-02-28

This article has been peer reviewed and published immediately upon acceptance.

It is an open access article, which means that it can be downloaded, printed, and distributed freely, provided the work is properly cited.

Articles in "Folia Morphologica" are listed in PubMed. 
The relationship between the aggrecan VNTR polymorphism and its content in lumbar intervertebral discs

\title{
P. Pękala et al., Aggrecan VNTR polymorphism
}

P. Pękala ${ }^{1}$, D. Felkle ${ }^{2}$, K. Dykas², A. Jarosz ${ }^{3}$, P. Elnazir ${ }^{1}$, T. Konopka ${ }^{4}$, J.A. Walocha ${ }^{1}$, J. Dulinska-Litewka ${ }^{2}$

${ }^{1}$ Department of Anatomy, Jagiellonian University Medical College, Krakow, Poland

${ }^{2}$ Department of Biochemistry, Jagiellonian University Medical College, Krakow, Poland

${ }^{3}$ Malopolska Centre of Biotechnology, Jagiellonian University, Krakow, Poland

${ }^{4}$ Department of Forensic Medicine, Jagiellonian University Medical College, Krakow, Poland

Address for correspondence: P. Pękala, Department of Anatomy, Jagiellonian University Medical College, ul. Kopernika 12, 31-034 Kraków, Poland, e-mail: pekala.pa@gmail.com

\begin{abstract}
Background: There is a specific polymorphism of the ACAN gene called the VNTR (variable number of tandem repeats), which is particularly interesting in the light of the development of intervertebral disc pathology and associated low back pain.
\end{abstract}


Materials and methods: The nucleus pulposus specimens were harvested from the L5/S1 intervertebral discs. The aggrecan content was determined using enzyme-linked immunosorbent assay (ELISA). Moreover, the VNTR polymorphism in the ACAN gene was evaluated.

Results: The genotyping of VNTR polymorphism in ACAN gene was successful in 94 tissue samples (48 homozygotes and 46 heterozygotes). The alleles were divided into four groups, in accordance with the number of tandem repeats in the ACAN gene. No difference between groups in the mean aggrecan mass nor in the mean degree of tissue moisture was observed.

Conclusions: No relationship between the ACAN gene VNTR polymorphism and the aggrecan content was observed in studied Caucasian cadavers. Such a relationship may be a more complex phenomenon and exists in other populations.

\section{Key words: aggrecan, intervertebral disc, morphology, risk factor}

\section{INTRODUCTION}

Low back pain is a common problem, which will eventually affect almost everyone in the population [1, 2]. It is diagnosed in patients across a variety of countries of differing socioeconomic status, profession and age groups [3]. While mechanical factors related to trauma and/or occupation such as heavy lifting may result in morphological damage to the intervertebral discs (IVDs) or contiguous soft tissues, many other risk factors of low back pain have been hypothesized; these include age, BMI, chronic diseases as well as genetic predisposition [2, 4]. Many studies have explored the significance of genetic polymorphisms in the pathogenesis of intervertebral disc degeneration (IDD) [5, 6]. One of the possible candidates is the ACAN gene (encoding aggrecan core protein). Its product constitutes the major proteoglycan of IVDs and the articular cartilage. The content of the aggrecan in IVDs has been shown to be crucial to 
their function [7]. A dense network of this proteoglycan forms aggregates which are osmotically active to draw water into the IVDs and cause an increase in the osmotic pressure of the tissue to allow for movement and the weight bearing functions of the spine [8]. It was reported that loss of this proteoglycan may play a role in the development of osteoarthritis and degenerative IVD disease [9]. There is a specific polymorphism of the ACAN gene called the VNTR (variable number of tandem repeats), which is particularly interesting. Precisely the humans were described to have between 11 to 33 repetitions of a specific 57 nucleotide sequence [10].

In a systematic study, Cong et al. suggested an interaction between obesity and this polymorphism in the development of IVD pathology in individuals of Han Chinese ethnicity, whilst another study by the same research group suggested a similar multiplicative relationship between VNTR, smoking and IDD [10, 11]. Therefore, the presence of this polymorphism may indeed impact the robust, weight-bearing functions of the spine. Moreover, the meta-analysis performed by Gu et al. suggested the increased risk of IDD related to presence of shorter (13-25 repetitions) compared with the normal, longer alleles in the ACAN gene. Such a relationship was found only in Asian populations, however not in Europeans [12]. Therefore, the aim of this study was to attempt to investigate the possible relationship between the VNTR polymorphism in the gene encoding aggrecan and its content within the Caucasian donor’s IVDs using accurate molecular methods.

\section{MATERIALS AND METHODS}

Sample preparation

The specimens were harvested from the L5/S1 IVDs collected from the 100 male cadaveric donors of age between 18 and 80. Additionally, the following exclusion criteria were used: ankylosing spondylitis, visible injury or/and surgery to the spine or such 
history. The mean age of the donors was 42.09 \pm 12.29 (all male Caucasian cadavers) [13].

After harvesting, all tissue samples were shattered manually using a scalpel into pieces of size $\approx 1 \mathrm{~mm}$ in every dimension. Then, the specimens were transferred to test tubes which were then weighed. Sample masses were calculated using the equation: weight of tube with sample - weight of empty tube = weight of sample.

\section{Solubilization}

The solution of 4M guanidine hydrochloride (Sigma-Aldrich, G3272-100G), 1M sodium acetate (Sigma-Aldrich, S2889-250G), Triton 2\% (Sigma-Aldrich, X100-1L) and protease inhibitor cocktail (Sigma-Aldrich, P8340-1ML) was added to the tissue samples homogenized as described above. Then specimens were processed at $4^{\circ} \mathrm{C}$ overnight on the laboratory shaker which was set on gentle shaking. After the solubilization, test tubes were centrifuged at $3000 \mathrm{rpm}$ for $10 \mathrm{~min}$ to obtain a supernatant which was transferred to new test tubes and was stored at $-20^{\circ} \mathrm{C}$.

\section{Enzyme-linked immunosorbent assay (ELISA)}

The temperature of the supernatant was raised to $\approx 20^{\circ} \mathrm{C}$. Then, the solutions were diluted (2-10 times) in fresh tubes with deionized water (Milli-Q Millipore device) to acquire concentrations in the range of detection of the method. ELISA was performed in accordance with the protocol provided by the manufacturer (RayBiotech, ELH-ACAN1). Then, the absorbance was assessed at 450nm using plate reader (Bio-TEK Synergy HT). The aggrecan concentration was established based on the mean of two separate measurements according to the standard curve constructed for each plate.

Genetic polymorphism in ACAN gene 
DNA was extracted from samples using Xpure Genomic Mini kit (A\&amp;A Biotechnology, Gdansk) according to the protocol recommended by the manufacturer. Purity of obtained DNA isolates was assessed using NanoDrop spectrophotometer (Thermo Scientific, Waltham, MA) and DNA concentration was measured with Qubit fluorometer (Thermo Scientific, Waltham, MA). PCR amplification of the CS1 domain in aggrecan gene was performed using the specific primers (sense primer: 5'TAGAGGGCTCTGCCTCTGGAGTTG-3’ and anti-sense primer: 5’AGGTCCCCTACCGCAGAGGTAGAA-3’). PCR reaction mixture consisted of: 1X Gold Buffer, $1.5 \mathrm{mM} \mathrm{MgCl}$ 2, $0.25 \mathrm{mM}$ of dNTPs, $0.6 \mu \mathrm{M}$ of each primer, $50 \mathrm{ng}$ of DNA, 1 U of AmpliTaq Gold DNA Polymerase (Applied Biosystems, Foster City, CA) and DNase-free water up to $25 \mu \mathrm{l}$. PCR was performed on C1000 Touch Thermal Cycler (Bio-Rad Laboratories, Hercules, CA) using following temperature profile: $95^{\circ} \mathrm{C}$ for 4 min, 33 cycles of $30 \mathrm{~s}$ at $95^{\circ} \mathrm{C}, 30 \mathrm{~s}$ at $63^{\circ} \mathrm{C}, 2 \mathrm{~min}$ at $72^{\circ} \mathrm{C}$, and final extension at $72^{\circ} \mathrm{C}$ for 10 min. PCR products were analyzed for their size on 2100 Bioanalyzer (Agilent) utilizing High Sensitivity DNA Assay. Number of repeats in the VNTR region was determined by comparison of the amplicon length. The alleles were divided into four groups, in accordance with the number of tandem repeats in the ACAN gene (I: A11-25; II: A26-27; III: A28-30; IV: A31-33).

\section{Statistical analysis}

Statistical analysis and data visualization were conducted using R Software (version 1.2.5042). For group comparison performance, Kruskal-Wallis test was applied, according to its assumptions. The correlation between variables was computed using Spearman's method. The significance level was set to 0.05 .

\section{Ethics}


The study protocol was accepted by the local bioethical committee. This study was performed in accordance with the principles of the Declaration of Helsinki and its later amendments.

\section{RESULTS}

In this study, the genotyping of VNTR polymorphism in ACAN gene was successful in 94 tissue samples (48 homozygotes and 46 heterozygotes). The distribution of the VNTR alleles was in accordance with the Hardy-Weinberg equilibrium [Figure 1A]. No difference between allele groups in the mean aggrecan mass nor in the mean degree of tissue moisture was observed [Table I]. Consistently, no correlation between aggrecan mass and the water content $(\mathrm{p}$-value $=0.173$ ) was found. The distribution of aggrecan mass, considering the degree of moisture and allele type is shown in Figure 1B.

\section{DISCUSSION}

To the best of the authors knowledge, it was the first human study analyzing the influence of the VNTR polymorphism in ACAN gene on the concentration of the aggrecan in the IVDs nucleus pulposus in Caucasian sample. The main goal was to investigate this hypothesized relationship using objective and accurate molecular methods.

Noteworthy is the fact that many case-control studies on VNTR polymorphism in ACAN gene and the IDD have been performed in the past, reporting frequently contradicting results [10-12]. Although they brought a plethora of valuable data, the significant bias likely existed due to uncontrolled factors contributing to the final development of the IDD, which was the main outcome based on what the cases and controls were allocated.

It is well-known that the low back pain symptoms are related not only to the pathology of the IVD, but also to morphological changes in the surrounding tissues such as lumbar facet joints, etc. [14]. Therefore, as it was shown in the literature that aggrecan 
concentration decreases in IVD with degeneration and aging, in this study it was decided to analyze this objective and isolated outcome, which is also the most logical causative result of the ACAN gene altered expression $[15,16]$.

The results of this study showed no relationship between the presence of short alleles and the aggrecan concentration in the nucleus pulposus of the L5/S1 IVDs. Interestingly, the meta-analysis performed by $\mathrm{Gu}$ et al., found a relationship between the IDD and the presence of the shorter alleles of the ACAN gene in Asian population, but reached no statistical significance pooling European samples [12]. Together with our observations it is likely that such phenomenon may be ethnic specific and/or other cofactors may play a significant role.

Moreover, the recent systematic study performed by Cong et al. found that the A21 allele (with 21 repetitions of a specific 57 nucleotide sequence) in ACAN gene is overexpressed in patients with IDD in their pooled sample. Interestingly, such a relationship was not identified for the rest of shorter and any other alleles [17]. However, in our study this allele had no representation among studied individuals. One must remember that considering our sample size, prevalence of particular alleles is relatively low and such comparisons require testing on numerous populations. Interestingly, the specimens from donors with the shortest alleles (A11-A25) were found to have lower water content than the IVDs from the other three groups (72.7\% vs. 76.0\%), however this difference was not significant. This observation may suggest that despite the VNTR in the ACAN gene does not influence the aggrecan dry mass in the nucleus pulposus of the lumbar IVD, it may alter its water attracting function. However, such a hypothesis cannot be confirmed by this study and requires further well-designed research.

Our study is limited by the uniform sex and ethnicity of the donors. However, it was designed to establish possible association in the Caucasian male population. Further, large studies on different populations are required in the future. The other limitation is a number of specimens included. However, it is challenging to perform larger cadaveric 
studies, and it is important that the results of this study can be pooled in systematic studies in the future to obtain more statistical power.

\section{CONCLUSIONS}

In summary, no relationship between the ACAN gene VNTR polymorphism and the aggrecan content was observed in studied Caucasian cadavers. Such a relationship may be a more complex phenomenon and exist in other populations.

\section{Acknowledgements}

We would like to acknowledge all the donors and their families, whose contribution allowed us to conduct this research. This research was supported by governmental funds for research in 2016-2021 (Polish Ministry of Science and Higher Education, Diamond Grant).

Conflict of interest: None declared

\section{REFERENCES}

[1] Hoy D, Brooks P, Blyth F, Buchbinder R. The Epidemiology of low back pain. Best Pract Res Clin Rheumatol. 2010 Dec;24(6):769-81. doi: 10.1016/j.berh.2010.10.002. PMID: 21665125.

[2] Balagué F, Mannion AF, Pellisé F, Cedraschi C. Non-specific low back pain. Lancet. 2012 Feb 4;379(9814):482-91. doi: 10.1016/S0140-6736(11)60610-7. Epub 2011 Oct 6. PMID: 21982256.

[3] Gleinert-Rożek MŁ, Kosiński A, Kaczyńska A, Zajączkowski M, Kuta W, Kamiński R, Piwko G. Metric analysis of the lumbar region of human vertebral column. 
Folia Morphol (Warsz). 2020;79(4):655-661. doi: 10.5603/FM.a2020.0008. Epub 2020 Feb 5. PMID: 32020579.

[4] Will JS, Bury DC, Miller JA. Mechanical Low Back Pain. Am Fam Physician. 2018 Oct 1;98(7):421-428. PMID: 30252425.

[5] Pekala PA, Henry BM, Taterra D, Piwowar M, Vikse J, Tubbs RS, Tomaszewski KA. FokI as a genetic factor of intervertebral disc degeneration: A PRISMA-compliant systematic review of overlapping meta-analyses. J Clin Neurosci. 2019 Feb;60:36-43. doi: 10.1016/j.jocn.2018.09.028. Epub 2018 Oct 9. PMID: 30309807.

[6] Biczo A, Szita J, McCall I, Varga PP; Genodisc Consortium, Lazary A.

Association of vitamin D receptor gene polymorphisms with disc degeneration. Eur Spine J. 2020 Mar;29(3):596-604. doi: 10.1007/s00586-019-06215-7. Epub 2019 Nov 25. Erratum in: Eur Spine J. 2020 Jan 14;: PMID: 31768839.

[7] Roughley P, Martens D, Rantakokko J, Alini M, Mwale F, Antoniou J. The involvement of aggrecan polymorphism in degeneration of human intervertebral disc and articular cartilage. Eur Cell Mater. 2006 Jan 18;11:1-7; discussion 7. PMID: 16425147.

[8] Urban JP, Roberts S. Degeneration of the intervertebral disc. Arthritis Res Ther. 2003;5(3):120-130. doi:10.1186/ar629

[9] Maldonado M, Nam J. The role of changes in extracellular matrix of cartilage in the presence of inflammation on the pathology of osteoarthritis. Biomed Res Int. 2013;2013:284873. doi:10.1155/2013/284873

[10] Cong L, Zhu Y, Pang H, Guanjun TU. The interaction between aggrecan gene VNTR polymorphism and obesity in predicting incident symptomatic lumbar disc herniation. Connect Tissue Res. 2014 Oct-Dec;55(5-6):384-90. doi: 10.3109/03008207.2014.959117. Epub 2014 Sep 22. PMID: 25188217. [11] Cong L, Pang H, Xuan D, Tu G. The interaction between aggrecan gene VNTR polymorphism and cigarette smoking in predicting incident symptomatic intervertebral disc degeneration. Connect Tissue Res. 2010 Oct;51(5):397-403. doi: 10.3109/03008200903564455. PMID: 20367118. 
[12] Gu J, Guan F, Guan G, Xu G, Wang X, Zhao W, Ji Y, Yan J. Aggrecan variable number of tandem repeat polymorphism and lumbar disc degeneration: a meta-analysis. Spine (Phila Pa 1976). 2013 Dec 1;38(25):E1600-7. doi:

10.1097/BRS.0000000000000012. PMID: 24296484.

[13] Pękala P, Taterra D, Krupa K, Paziewski M, Wojciechowski W, Konopka T, Walocha JA, Tomaszewski KA. Correlation of morphological and radiological characteristics of degenerative disc disease in lumbar spine: a cadaveric study. Folia Morphol (Warsz). 2021 Apr 26. doi: 10.5603/FM.a2021.0040. Epub ahead of print. PMID: 33899206.

[14] Kapetanakis S, Gkantsinikoudis N. Anatomy of lumbar facet joint: a comprehensive review. Folia Morphol (Warsz). 2021;80(4):799-805. doi: 10.5603/FM.a2020.0122. Epub 2020 Oct 21. PMID: 33084010.

[15] Antoniou J, Steffen T, Nelson F, Winterbottom N, Hollander AP, Poole RA, Aebi M, Alini M. The human lumbar intervertebral disc: evidence for changes in the biosynthesis and denaturation of the extracellular matrix with growth, maturation, ageing, and degeneration. J Clin Invest. 1996 Aug 15;98(4):996-1003. doi: 10.1172/JCI118884. PMID: 8770872; PMCID: PMC507515.

[16] Pearce, R.H., J.P. Thompson, G.M. Bebault, and B. Flak. 1991. Magnetic resonance imaging reflects changes of ageing degeneration in the human intervertebral disc. J. Rheumatol. 18(Suppl. 27):42-43.

[17] Cong L, Tu G, Liang D. A systematic review of the relationship between the distributions of aggrecan gene VNTR polymorphism and degenerative disc disease/osteoarthritis. Bone Joint Res. 2018 May 5;7(4):308-317. doi: 10.1302/20463758.74.BJR-2017-0207.R1. PMID: 29922449; PMCID: PMC5987698. 
Table I. Mean aggrecan mass per dry tissue and mean degree of moisture by number of tandem repeats in the ACAN gene. *Kruskal-Wallis test

\begin{tabular}{|c|c|c|c|c|}
\hline Alleles & $\begin{array}{l}\text { Mean aggrecan } \\
\text { mass per dry tissue } \\
\text { [ng/g] (SD) }\end{array}$ & p-value* & $\begin{array}{l}\text { Mean degree of } \\
\text { moisture (SD) }\end{array}$ & p-value* \\
\hline A11- 25 & $55.83(6.56)$ & \multirow[t]{4}{*}{0.1515} & $0.73(0.05)$ & \multirow[t]{4}{*}{0.6264} \\
\hline A26 - 27 & $41.60(17.96)$ & & $0.77(0.04)$ & \\
\hline A28 - 30 & $51.76(38.18)$ & & $0.76(0.04)$ & \\
\hline A31 - 33 & $35.12(17.94)$ & & $0.75(0.05)$ & \\
\hline
\end{tabular}

Figure 1. A. The histogram of alleles frequencies; B. The distribution of aggrecan mass, considering the degree of moisture and allele type

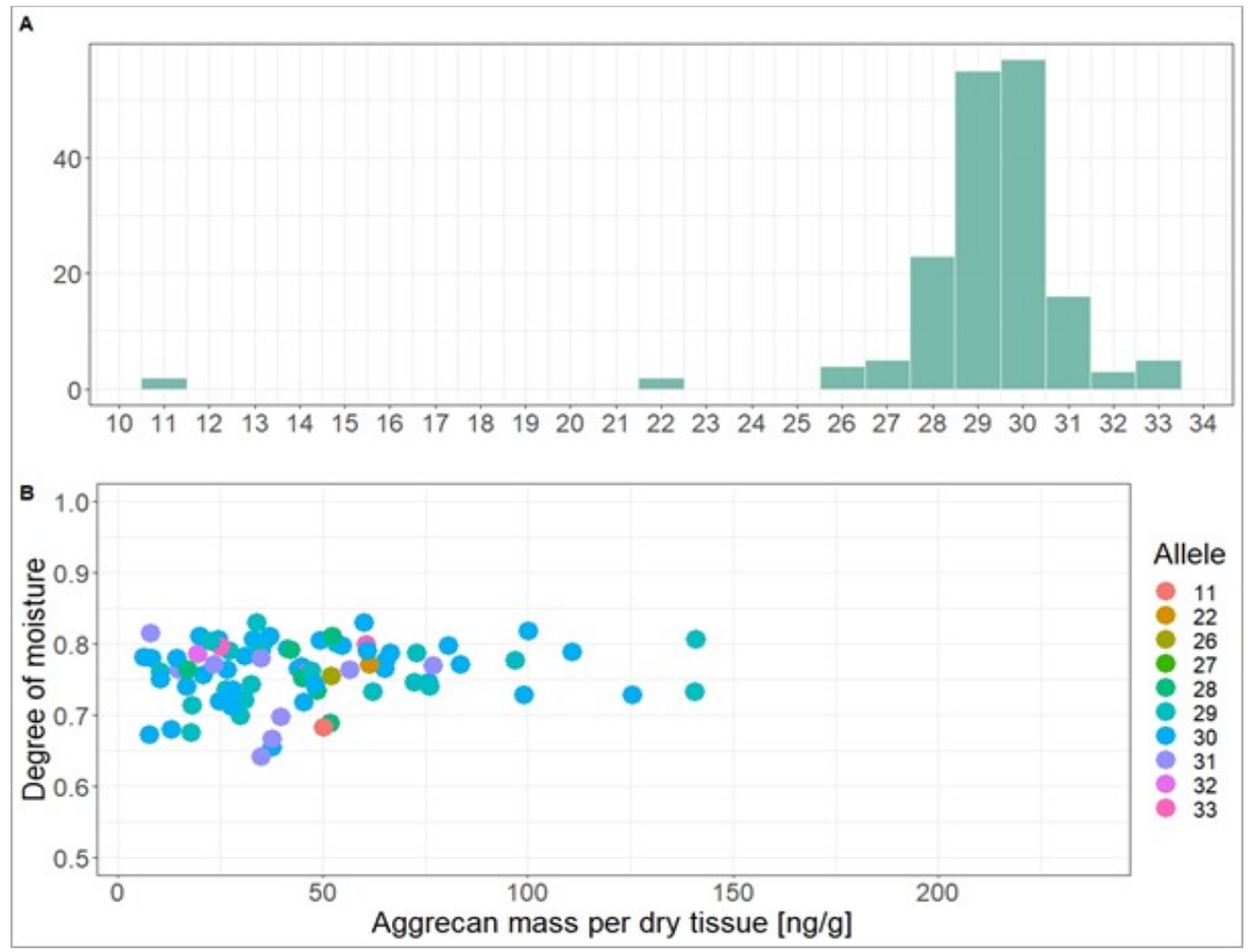

